\title{
Morphological characteristics of the proximal femur in elderly patients with hip fractures: a case-control study
}

Jixing Fan ${ }^{1}$, Ning $\mathrm{Li}^{2}$, Xiaofeng Gong ${ }^{2}$, Liang $\mathrm{He}^{2}$

${ }^{1}$ School of Clinical Medicine, Tsinghua University, Beijing, China

${ }^{2}$ Department of Orthopedics and Traumatology, Beijing Jishuitan Hospital, Beijing, China

Submitted: 16 June 2017

Accepted: 17 October 2017

Arch Med Sci Civil Dis 2017; 2: e161-e167

DOI: https://doi.org/10.5114/amscd.2017.71514

Copyright $\odot 2017$ Termedia \& Banach

\section{Abstract}

Introduction: Owing to the diverse design, measurement methods and ethnic differences, the influence of the proximal femur geometry on hip fractures is still unclear. Therefore, this study aimed to investigate morphological characteristics of the proximal femur in senile patients with hip fractures on three-dimensional images.

Material and methods: One hundred and sixteen women and 38 men with hip fractures were included in the fracture group. The control group included 74 women and 63 men. The geometrical parameters of the proximal femur were measured after three-dimensional reconstruction. The femoral neck width (FNW), femoral neck length (FNL), femoral head height $(\mathrm{FHH})$, femoral head diameter (FHD), neck shaft angle (NSA) and offset were measured and statistically analyzed.

Results: The NSA in fracture cases was significantly larger than controls in both men and women ( 130.18 vs. $126.93, p=0.001 ; 131.07$ vs. 128.68 , $p<0.001$, respectively). Moreover, a lower total hip bone mineral density (BMD) in fracture cases was found in both sexes ( 0.725 vs. $0.812, p=0.001$; 0.743 vs. $0.830, p<0.001$, respectively). In multiple logistic regression analysis, a larger NSA and a lower total hip BMD were independent predictors for hip fractures in both men and women $(O R=1.143$ and $1.171, p=0.010$ and 0.016 , respectively). However, the FNL was an independent predictor for hip fractures only for women $(\mathrm{OR}=1.201,95 \% \mathrm{Cl}: 1.106-1.305, p<0.001)$. Conclusions: A larger NSA and a lower BMD were independent predictors for hip fractures of senile patients in both sexes. Moreover, a longer FNL was an independent risk factor for patients with hip fractures in women. As a result, we hypothesized that the geometrical measurement of the proximal femur on three-dimensional images might be appropriate.

Key words: hip fractures, proximal femur, geometry.

\section{Introduction}

The majority of fragility fractures in elderly patients occur as a result of only moderate or minimal trauma, such as a fall from a standing height. The main reason is osteoporosis, which is characterized by low bone mass and destruction of the bone microarchitecture, resulting in increased fragility [1]. Hip fracture in elderly patients is a kind of osteoporotic fractures, which is becoming a healthcare problem with the increase of fracture incidence owing to the increasing aged population $[2,3]$. Therefore, it is of great concern to investigate predictors for the occurrence of hip fractures.

\author{
Corresponding author: \\ Liang He MD \\ Department \\ of Orthopedics \\ and Traumatology \\ Beijing Jishuitan Hospital \\ No. 31 Xinjiekou East St \\ Xicheng District \\ 100035 Beijing, China \\ Phone: +861058516549 \\ Fax: +86 1058516549 \\ E-mail: heliang_jst@163.com
}


Several risk factors have been found to be associated with hip fractures, such as older age, history of fracture, low bone mineral density (BMD), current smoking, and dementia [4-6]. Currently, the proximal femur BMD measured by dual energy $X$-ray absorptiometry (DXA) is considered as the gold standard for the prediction of hip fractures [7]. However, one study revealed that up to $50 \%$ of fragile fractures did not have osteoporosis as demonstrated by BMD [8]. Furthermore, several studies revealed that the simple measurement of the proximal femur geometry could predict the occurrence of hip fractures independently of BMD [9, 10]. At present, the proximal femur geometry had been extensively investigated to predict the occurrence of hip fractures in elderly patients [11-13].

However, different measurement methods, ethnic differences and diverse study design have resulted in conflicting results about the measurement of the proximal femur geometry $[14,15]$. Moreover, the majority of measurements were performed on two-dimensional images, such as DXA or X-ray radiograph, which might be influenced by patients' position. Furthermore, studies on hip geometry for elderly Asians are very rare. Therefore, we applied a new method for the measurement, which was performed on computed tomography (CT) images after three-dimensional (3D) reconstruction in order to predict hip fractures more accurately. This could minimize the effect of patients' position on measurements. This study aimed to investigate morphological characteristics of the proximal femur in senile patients with hip fractures.

\section{Material and methods}

\section{Subjects}

In this retrospective case-control study, 216 patients aged 65 years or older with osteoporotic hip fractures were included in this study from May 2015 to May 2016. All fractures were caused by low-energy injury. The excluded criteria were as follows: 1) Patients combined with diseases affecting the bone metabolism (osteomalacia, Paget disease, or primary hyperparathyroidism); 2) high-energy injury; 3) patients who were taking drugs or had a history of drug use, such as teriparatide, bisphosphonates; 4) pathological fractures (secondary to the tumor or primary hyperparathyroidism). Eventually, 154 patients were included in this study, which consisted of 38 male patients and 116 female patients. The research was permitted by the ethics committee of the institute.

\section{Measurements}

A TOSHIBA CT scanner (TOSHIBA, Tokyo, Japan) was applied to perform the CT scan for all patients after being admitted to the hospital. The CT was set to $120 \mathrm{kVp}$ and $125 \mathrm{mAs}$ to produce an image matrix of $512 \times 512$ pixels. The thickness of each slice was $1.0 \mathrm{~mm}$. The healthy leg was placed in the position with $0^{\circ}$ of extension during imaging. All CT scan data were numbered and downloaded to a personal computer with the Digital Imaging and Communications in Medicine (DICOM) format. Then $\mathrm{CT}$ images were imported into the reverse engineering software Mimics 17.0 (Materialise, Belgium) software for three dimensional reconstruction and geometrical measurement.

The 3D proximal femur model of the healthy leg was created after mask creation, region growing and calculation. Then the femoral head was separated and simulated to a ball. We regarded the center of the ball as the center of the femoral head [16]. The transverse slice at the level of apex of the lesser trochanter was defined as slice TO. Slices $10 \mathrm{~mm}$ and $30 \mathrm{~mm}$ distal to the end of the lesser trochanter were defined as slice T10 and slice T30. Then a circle was drawn in slice T10 and T30 which was best fit with the inner contour of a section plane. Then a line was drawn along the center of slices T10 and T30. The line was defined as the central axis of the proximal medullary canal (Figure 1). The plane defined by the central axis of the proximal femur and the center of the femoral head was named as the central coronal plane of the proximal femur [17]. Geometrical parameters were measured on this plane. Parameters were as follows: 1) Femoral neck width (FNW): the narrowest femoral neck width; 2) Femoral neck length (FNL): a line was drawn through the midpoint of the FNW and the center of the femoral head, which intersected with the cortex of the proximal femur and the femoral head at a point. This line was defined as the central axis of the femoral neck. The length between the above two points was defined as the FNL; 3) Femoral head height $(\mathrm{FHH})$ : the vertical distance between the center of the femoral head and the slice T0; 4) Femoral head diameter (FHD); 5) Neck-shaft angle (NSA): the angle was formed between the axis of the femoral neck and the axis of the proximal femur; 6) Offset: the perpendicular distance from the centre of the femoral head to the central axis of the proximal medullary canal (Figure 2).

\section{Bone mineral density}

Areal BMD $\left(\mathrm{g} / \mathrm{cm}^{2}\right)$ of the total hip was measured by dual-energy X-ray absorptiometry with a Lunar DPX device (GE; Fairfield, Connecticut, USA) in the array (fan beam) mode.

\section{Statistical analysis}

For continuous variables, the independent Student's $t$-test was used to compare data between 

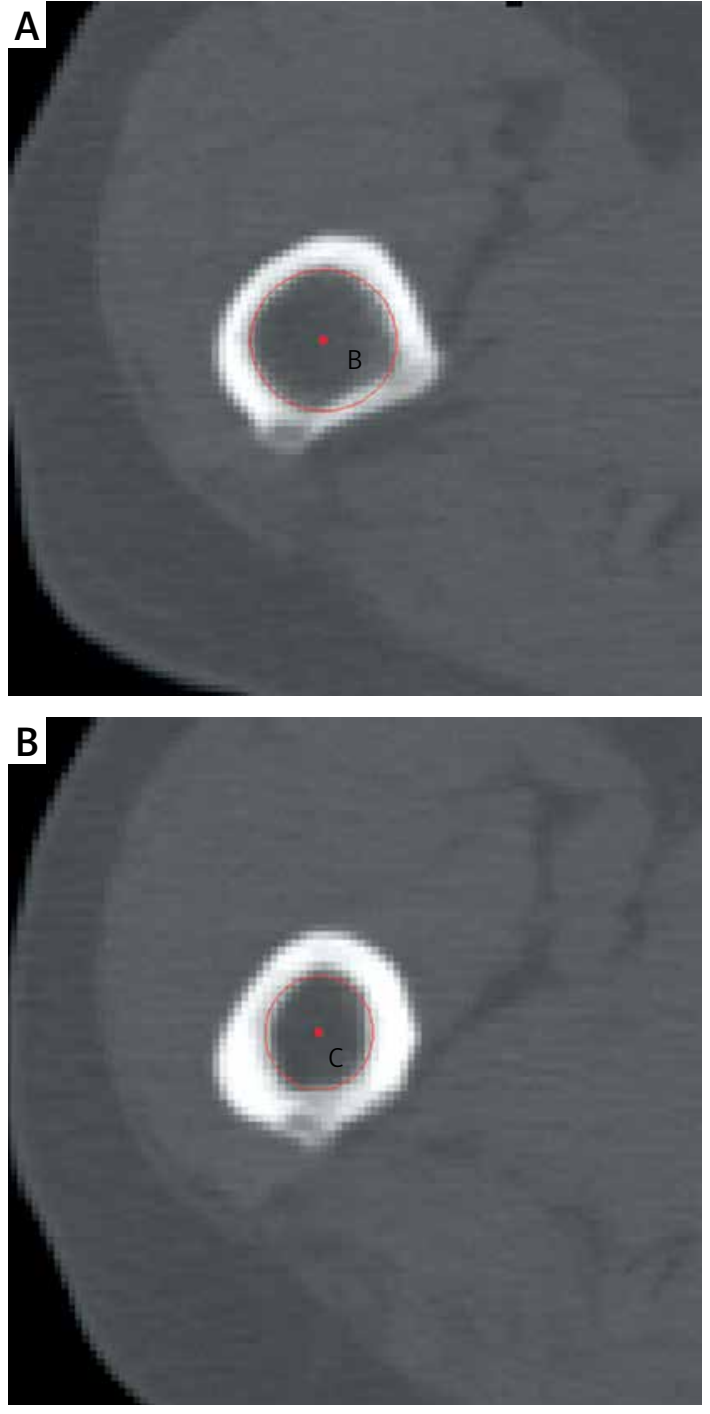

groups if the data showed a normal distribution; otherwise the Mann-Whitney test was used. For qualitative data, the $\chi^{2}$ test was used. Then the multiple logistic regression analysis was applied to evaluate the independent risk factor for hip fractures. Furthermore, receiver operating curve (ROC) analysis was performed to evaluate the predictability of each index. The value of area under the curve (AUC) was calculated. A P-value $<0.05$ was considered as statistically significant, and all tests were two-sided. The SPSS 17.0 software was used for statistical analysis (SPSS, Chicago, Illinois, USA).

\section{Results}

Of the 154 patients with hip fractures, 38 (25\%) patients were men and 116 (75\%) cases were women. Male patients in the fracture group were significantly older than patients in the control group (76.76 vs. $71.97, p=0.004$ ), while in female patients, there was no significant difference in age (79.96 vs. 78.91, $p=0.314$ ). The NSA
C

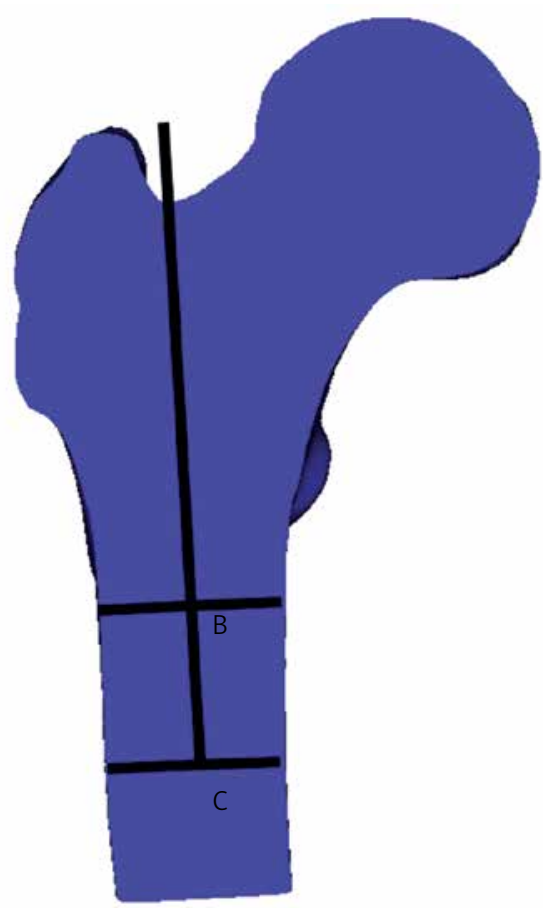

Figure 1. Definition of the femoral shaft axis of the proximal femur. A - The transverse slice at the level of $10 \mathrm{~mm}$ distal to the end of the lesser trochanter (T10). B - The transverse slice at the level of $30 \mathrm{~mm}$ distal to the end of the lesser trochanter (T30). C-B, the center of the slice T10; $C$ - the center of the slice T30; $\mathrm{B}-\mathrm{C}$, the femoral shaft axis of the proximal femur

in fracture cases was significantly larger than that in controls in both men and women (130.18 vs. $126.93, p=0.001 ; 131.07$ vs. $128.68, p<0.001$, respectively). In addition, no differences in FNL were found between the fracture group and control group in male patients (91.72 vs. 91.41, $p=$ 0.790 ), whereas the FNL in the fracture group was significantly longer than that in the control group in female patients (88.86 vs. 85.59, $p<0.001$ ). Moreover, a lower total hip BMD in fracture cases was found than in control cases in both sexes ( 0.725 vs. $0.812, p=0.001 ; 0.743$ vs. $0.830, p<$ 0.001 , respectively). The other parameters such as height, weight, body mass index (BMI), FNW, FHH, FHD and offset did not reach statistically significant differences $(p>0.05)$. The details are shown in Tables I and II.

In multiple logistic regression analysis, a larger NSA was an independent predictor for hip fractures in both men and women after adjusting for age and other confounding factors $(O R=1.143$, $95 \% \mathrm{Cl}: 1.027-1.272, p=0.014 ; \mathrm{OR}=1.171$, 


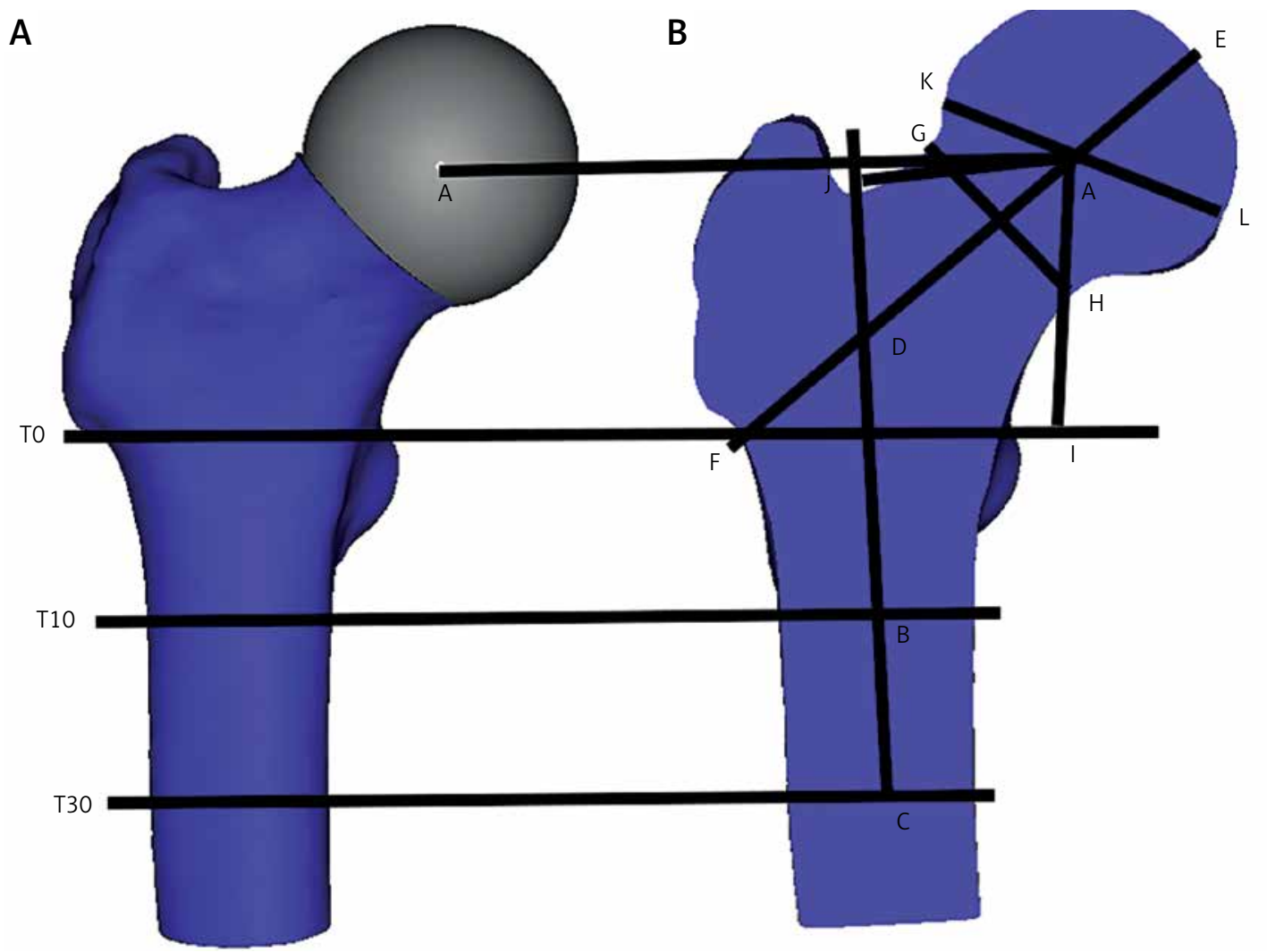

Figure 2. Geometrical measurement of the proximal femur. TO - The transverse slice at the level of the apex of the lesser trochanter; $\mathrm{G}-\mathrm{H}$ - femoral neck width; E-F - femoral neck length; A-I - femoral head height; $\mathrm{K}-\mathrm{L}$ - femoral head diameter; A-D-C - neck shaft angle. A-J - offset

Table I. Demographic characteristics and geometric parameters in men

\begin{tabular}{|lccll|}
\hline Characteristics & $\begin{array}{c}\text { Fracture group } \\
(n=38)\end{array}$ & $\begin{array}{c}\text { Control group } \\
(n=63)\end{array}$ & $P$-value & Value of AUC \\
\hline Age [years] & $76.76 \pm 7.34$ & $71.97 \pm 8.25$ & 0.004 & 0.679 \\
\hline Height [cm] & $169.03 \pm 4.11$ & $167.39 \pm 5.09$ & 0.098 & 0.594 \\
\hline Weight $[\mathrm{kg}]$ & $69.95 \pm 7.53$ & $68.73 \pm 7.38$ & 0.427 & 0.557 \\
\hline BMI $\left[\mathrm{kg} / \mathrm{m}^{2}\right]$ & $24.45 \pm 2.12$ & $24.51 \pm 2.22$ & 0.895 & 0.502 \\
\hline Smokers & 8 & 15 & 0.749 & \\
\hline Drinkers & 12 & 21 & 0.855 & 0.536 \\
\hline FNW $[\mathrm{mm}]$ & $33.39 \pm 2.63$ & $33.09 \pm 2.09$ & 0.526 & 0.600 \\
\hline FNL $[\mathrm{mm}]$ & $91.72 \pm 6.59$ & $91.41 \pm 4.85$ & 0.790 & 0.556 \\
\hline FHH $[\mathrm{mm}]$ & $36.19 \pm 5.25$ & $37.11 \pm 4.82$ & 0.373 & 0.535 \\
\hline FHD $[\mathrm{mm}]$ & $48.05 \pm 3.08$ & $48.15 \pm 1.92$ & 0.838 & 0.667 \\
\hline NSA & $130.18 \pm 5.15$ & $126.93 \pm 4.45$ & 0.001 & 0.593 \\
\hline Offset $[\mathrm{mm}]$ & $32.22 \pm 2.91$ & $33.53 \pm 3.93$ & 0.078 & 0.655 \\
\hline Total hip BMD $\left[\mathrm{g} / \mathrm{cm}^{2}\right]$ & $0.725 \pm 0.131$ & $0.812 \pm 0.112$ & 0.001 & \\
\hline
\end{tabular}

$A U C$ - area under the curve, BMI - body mass index, FNW - femoral neck width, FNL - femoral neck length, FHH - femoral head height, FHD - femoral head diameter, NSA - neck shaft angle, BMD - bone mineral density.

$95 \% \mathrm{Cl}: 1.073-1.277, p<0.001$, respectively). However, age was an independent risk factor only for men $(\mathrm{OR}=1.062,95 \% \mathrm{Cl}: 1.003-1.125, p=0.038)$.
Moreover, FNL was an independent predictor for hip fractures only for women $(\mathrm{OR}=1.201,95 \% \mathrm{Cl}$ : 1.106-1.305, $p<0.001)$. With respect to total hip 
Table II. Demographic characteristics and geometric parameters in women

\begin{tabular}{|lcccc|}
\hline Characteristics & $\begin{array}{c}\text { Fracture group } \\
(n=116)\end{array}$ & $\begin{array}{c}\text { Control group } \\
(n=74)\end{array}$ & $P$-value & Value of AUC \\
\hline Age [years] & $79.96 \pm 6.68$ & $78.91 \pm 7.49$ & 0.314 & 0.537 \\
\hline Height [cm] & $160.38 \pm 4.76$ & $159.79 \pm 4.70$ & 0.410 & 0.534 \\
\hline Weight [kg] & $59.86 \pm 8.51$ & $59.66 \pm 8.82$ & 0.879 & 0.519 \\
\hline BMI [kg/m²] & $23.25 \pm 3.01$ & $23.32 \pm 2.92$ & 0.882 & 0.501 \\
\hline Smokers & 12 & 7 & 0.843 & \\
\hline Drinkers & 15 & 10 & 0.908 & 0.534 \\
\hline FNW [mm] & $29.53 \pm 2.16$ & $29.34 \pm 1.91$ & 0.521 & 0.674 \\
\hline FNL [mm] & $88.86 \pm 5.77$ & $85.59 \pm 4.57$ & $<0.001$ & 0.557 \\
\hline FHH [mm] & $33.07 \pm 4.22$ & $32.08 \pm 3.54$ & 0.095 & 0.512 \\
\hline FHD [mm] & $43.76 \pm 2.21$ & $43.84 \pm 2.33$ & 0.810 & 0.655 \\
\hline NSA & $131.07 \pm 4.72$ & $128.68 \pm 3.24$ & $<0.001$ & 0.527 \\
\hline Offset [mm] & $29.87 \pm 3.48$ & $29.89 \pm 2.59$ & 0.944 & 0.654 \\
\hline Total hip BMD [g/cm²] & $0.743 \pm 0.140$ & $0.830 \pm 0.165$ & $<0.001$ & \\
\hline
\end{tabular}

AUC - area under the curve, BMI - body mass index, FNW - femoral neck width, FNL - femoral neck length, FHH - femoral head height, $F H D$ - femoral head diameter, NSA - neck shaft angle, BMD - bone mineral density.

Table III. Multivariable logistic regression analysis for predictors associated with hip fracture types in both sexes

\begin{tabular}{|lcccccc|}
\hline Predictor & \multicolumn{3}{c}{ Men } & \multicolumn{3}{c|}{ Women } \\
\cline { 2 - 7 } & Adjusted OR & $95 \% \mathrm{Cl}$ & $P$-value* & Adjusted OR & $95 \% \mathrm{Cl}$ & $P$-value* \\
\hline Age & 1.062 & $1.003-1.125$ & 0.038 & 1.031 & $0.982-1.083$ & 0.221 \\
\hline FNL & 1.019 & $0.928-1.118$ & 0.692 & 1.201 & $1.106-1.305$ & $<0.001$ \\
\hline FNW & 1.182 & $0.963-1.450$ & 0.110 & 1.154 & $0.929-1.435$ & 0.196 \\
\hline FHD & 0.929 & $0.730-1.182$ & 0.549 & 0.778 & $0.651-0.931$ & 0.006 \\
\hline NSA & 1.143 & $1.027-1.272$ & 0.014 & 1.171 & $1.073-1.277$ & $<0.001$ \\
\hline Offset & 0.969 & $0.831-1.131$ & 0.691 & 1.028 & $0.908-1.164$ & 0.660 \\
\hline BMD & 0.010 & $0.000-0.652$ & 0.031 & 0.016 & $0.002-0.160$ & $<0.001$ \\
\hline
\end{tabular}

OR - odds ratio, FNL - femoral neck length, FNW - femoral neck length, FHD - femoral head diameter, NSA - neck shaft angle, BMD bone mineral density. ${ }^{*} T h e$ multivariable regression analysis used backward selection using the likelihood ratio test to assess significance.

$\mathrm{BMD}$, a lower BMD was independent risk factor for hip fractures in both sexes $(O R=0.010,95 \%$ $\mathrm{Cl}$ : 0.000-0.652, $p=0.031$; OR $=0.016,95 \% \mathrm{Cl}$ : $0.002-0.160, p<0.001$, respectively) (Table III).

According to ROC curves calculated for each parameter, age, NSA and total hip BMD had better AUC values in men $(0.679,0.667$ and 0.655 , respectively). In addition, the FNL, NSA and total hip BMD had better AUC values in women (0.674, 0.655 and 0.654 , respectively) (Tables I and II).

\section{Discussion}

Owing to the high rate of morbidity and mortality in hip fractures, it is necessary to predict the occurrence of hip fractures accurately. Various studies have demonstrated that the proximal femur geometry was associated with the occurrence of hip fractures in elderly patients [12, 18]. Similar to previous studies [10,13], a larger neck shaft angle and a longer femoral neck length were found to be independent predictors for hip fractures in both sexes. In addition, consistently with previous results, a lower hip BMD was an independent risk factor for the occurrence of hip fractures $[13,18]$.

Currently, the relationship between the NSA and hip fractures is still controversial $[12,18]$. One study revealed that acute NSA was found in the fracture group compared with the control group [18], while the reason for this was unclear in this study. In contrast, the majority of previous studies confirmed that a larger NSA was associated 
with the occurrence of hip fractures [19, 20]. Gomez Alonso et al. [13] reported that an increase of 1 standard deviation (SD) in the NSA was associated with an odds ratio of hip fractures of 2.45 in men and 3.48 in women. Ripamonti et al. [20] noted that the NSA was found to be associated with hip fracture risk in males, and the data indicated that the NSA was associated with femoral neck resistance to stress when falling on the greater trochanter. With the increase of the NSA, the bending moment at the femoral neck increased simultaneously and the impact force imposed on the femoral neck increased at the same time, which resulted in a greater probability of hip fracture [21, 22]. In addition, bone remodeling occurred at all stages of life, especially when the loading condition changed [23]. Some authors reported that negative correlations were found between NSA and cortical thickness, which indicated that bone tended to increase its strength by adapting to the loading condition $[24,25]$. However, the human bone had not adapted to the loading conditions of falling direction and the fracture might occur when people fell down [26]. In the present study, a larger NSA was found to be an independent predictor for hip fractures in both men and women. With an AUC value of 0.667 in men and 0.655 in women, the NSA was regarded as a better parameter for predicting hip fractures compared with other parameters in this study.

A longer femoral neck length was considered as an independent predictor for hip fractures in previous studies [9, 10, 27]. Leslie et al. [27] investigated 1020 hospitalized hip fractures and found that hip axis length was an independent risk factor for hip fractures when adjusted for fracture risk assessment tool and BMD in women. Another study revealed that hip axis length presented a positive and statistically significant correlation with hip fractures when analyzed in isolation and when adjusted for femoral neck BMD in Caucasian elderly Brazilian women [10]. The greater trochanter of the femur might extend further beyond the pelvis in people with a longer FNL than in those with a shorter FNL, causing hips with a longer FNL to be predisposed to fracture. In the present study, the FNL in the fracture group was significantly longer than the control group, and the FNL was an independent predictor for hip fractures after adjusting for age and other confounding factors in female patients. However, no significant difference was found between fracture and control groups in male patients. In general, men had a longer hip axis length than women. There was little evidence that men with hip fractures had a longer hip axis length than age-matched controls [28]. The body size difference might cause the discrepancy of FNL between men and women in the present study.

In the present study, a lower total hip BMD was an independent predictor for hip fractures in both men and women. It has been extensively demonstrated that patients with low bone mineral density of the proximal femur probably have high in cidence of hip fractures $[18,19]$. One study found that BMD at each measurement site of the femoral neck, trochanter and Ward's triangle in the fracture group was lower than the control group, and the results showed that the site-matched BMD was the best predictor of both femoral neck and trochanteric fractures [29]. Another study demonstrated that older men with low femoral neck BMD had a high risk of hip fractures [4]. Moreover, one study revealed that the measurement of the hip BMD could provide a strong risk indicator for fracture that was largely independent of sex [30]. In accordance with previous studies, a lower total hip BMD was found in the fracture group for both sexes. However, the other sites of BMD apart from total hip were not measured in this study, which might be one of the limitations of this study.

Various studies have demonstrated that older age was an independent predictor for hip fractures $[4,31]$. The possible reason might be that age influenced the distribution of BMD in the proximal femur, which was a strong predictor for osteoporotic fractures. In the present study, older age was an independent risk factor for hip fractures in men, while no significant difference was found in women. The reasons for this might be the small sample size. Therefore, a study with a larger sample is needed to verify the present results.

The greatest strength of this study was that the parameter was measured on a three-dimensional image, which would minimize the error. Moreover, the geometrical parameters were analyzed separately in men and women. In addition, there were a number of limitations in this study. Firstly, the sample was relatively small. Secondly, patients with hip fractures were not categorized according to fracture type, such as femoral neck fractures and intertrochanteric fractures. Thirdly, the study design was retrospective. Therefore, the results of this study should be evaluated with some caution and further studies with a larger sample size and prospective design are needed.

In conclusion, a larger NSA and a lower BMD were independent predictors for hip fractures of senile patients in both sexes. Moreover, a longer FNL was an independent risk factor for patients with hip fractures in women, whereas no statistically significant difference was found in men. In addition, the geometrical measurement of the proximal femur on three-dimensional images might be appropriate.

\section{Acknowledgments}

We are grateful to all caretakers and assistants who helped to collect data: Haonan Liu, Dongchen Yao and Qifei He. 


\section{Conflict of interest}

The authors declare no conflict of interest.

\section{References}

1. Consensus development conference: diagnosis, prophylaxis, and treatment of osteoporosis. Am J Med 1993; 94: 646-50.

2. Dash SK, Panigrahi R, Palo N, Priyadarshi A, Biswal M. Fragility hip fractures in elderly patients in Bhubaneswar, India (2012-2014): a prospective multicenter study of 1031 elderly ptients. Geriatr Orthop Surg Rehabil 2015; 6: 11-5.

3. Diamantopoulos AP, Rohde G, Johnsrud I, et al. Incidence rates of fragility hip fracture in middle-aged and elderly men and women in southern Norway. Age Ageing 2012; 41: 86-92.

4. Cauley JA, Cawthon PM, Peters KE, et al. Risk factors for hip fracture in older men: the osteoporotic fractures in men study (MrOS). J Bone Miner Res 2016; 31: 1810-9.

5. Lee SH, Chen IJ, Li YH, Fan Chiang CY, Chang CH, Hsieh PH. Incidence of second hip fractures and associated mortality in Taiwan: a nationwide population-based study of 95,484 patients during 2006-2010. Acta Orthop Traumatol Turc 2016; 50: 437-42.

6. Maravic M, Ostertag A, Urena P, Cohen-Solal M. Dementia is a major risk factor for hip fractures in patients with chronic kidney disease. Osteoporos Int 2016; 27: 1665-9.

7. Beck TJ, Ruff CB, Warden KE, Scott WW Jr, Rao GU. Predicting femoral neck strength from bone mineral data. A structural approach. Invest Radiol 1990; 25: 6-18.

8. Wainwright SA, Marshall LM, Ensrud KE, et al. Hip fracture in women without osteoporosis. J Clin Endocrinol Metab 2005; 90: 2787-93.

9. Faulkner KG, Cummings SR, Black D, Palermo L, Gluer CC, Genant HK. Simple measurement of femoral geometry predicts hip fracture: the study of osteoporotic fractures. J Bone Miner Res 1993; 8: 1211-7.

10. Frisoli A Jr, Paula AP, Pinheiro $M$, et al. Hip axis length as an independent risk factor for hip fracture independently of femural bone mineral density in Caucasian elderly Brazilian women. Bone 2005; 37: 871-5.

11. Dincel VE, Sengelen M, Sepici V, Cavusoglu T, Sepici B. The association of proximal femur geometry with hip fracture risk. Clin Anat 2008; 21: 575-80.

12. Han J, Hahn MH. Proximal femoral geometry as fracture risk factor in female patients with osteoporotic hip fracture. J Bone Metab 2016; 23: 175-82.

13. Gomez Alonso C, Diaz Curiel M, Hawkins Carranza F, Perez Cano R, Diez Perez A. Femoral bone mineral density, neck-shaft angle and mean femoral neck width as predictors of hip fracture in men and women. Osteoporos Int 2000; 11: 714-20.

14. Tang ZH, Yeoh CS, Tan GM. Radiographic study of the proximal femur morphology of elderly patients with femoral neck fractures: is there a difference among ethnic groups? Singapore Med J 2016; DOI: 10.11622/ smedj.2016148.

15. Imren Y, Sofu H, Dedeoglu SS, Desteli EE, Cabuk H, Kir MC. Predictive value of different radiographic parameters evaluating the proximal femoral geometry for hip fracture in the elderly: what is the role of the true moment arm? Arch Med Sci Civil Dis 2016; 1: 58-62.

16. Fu X, Xu GJ, Li ZJ, et al. Three-dimensional reconstruction modeling of the spatial displacement, extent and rotational orientation of undisplaced femoral neck fractures. Medicine (Baltimore) 2015; 94: e1393.

17. Liu S, Zuo J, Li Z, et al. Study of three-dimensional morphology of the proximal femur in developmental adult dysplasia of the hip suggests that the on-shelf modular prosthesis may not be an ideal choice for patients with Crowe type IV hips. Int Orthop 2017; 41: 707-13.

18. Lee DH, Jung KY, Hong AR, et al. Femoral geometry, bone mineral density, and the risk of hip fracture in premenopausal women: a case control study. BMC Musculoskelet Disord 2016; 17: 42.

19. Gnudi S, Sitta E, Pignotti E. Prediction of incident hip fracture by femoral neck bone mineral density and neckshaft angle: a 5-year longitudinal study in post-menopausal females. Br J Radiol 2012; 85: e467-73.

20. Ripamonti C, Lisi L, Avella M. Femoral neck shaft angle width is associated with hip-fracture risk in males but not independently of femoral neck bone density. Br J Radiol 2014; 87: 20130358.

21. Wang Q, Teo JW, Ghasem-Zadeh A, Seeman E. Women and men with hip fractures have a longer femoral neck moment arm and greater impact load in a sideways fall. Osteoporos Int 2009; 20: 1151-6.

22. Pulkkinen P, Eckstein F, Lochmuller EM, Kuhn V, Jamsa T. Association of geometric factors and failure load level with the distribution of cervical vs. trochanteric hip fractures. J Bone Miner Res 2006; 21: 895-901.

23. Frost HM. Skeletal structural adaptations to mechanical usage (SATMU): 1. Redefining Wolff's law: the bone modeling problem. Anat Rec 1990; 226: 403-13.

24. Rafferty KL. Structural design of the femoral neck in primates. J Hum Evol 1998; 34: 361-83.

25. Partanen J, Jamsa T, Jalovaara P. Influence of the upper femur and pelvic geometry on the risk and type of hip fractures. J Bone Miner Res 2001; 16: 1540-6.

26. Frost HM. Skeletal structural adaptations to mechanical usage (SATMU): 2. Redefining Wolff's law: the remodeling problem. Anat Rec 1990; 226: 414-22.

27. Leslie WD, Lix LM, Morin SN, et al. Hip axis length is a FRAX- and bone density-independent risk factor for hip fracture in women. J Clin Endocrinol Metab 2015; 100: 2063-70.

28. Center JR, Nguyen TV, Pocock NA, et al. Femoral neck axis length, height loss and risk of hip fracture in males and females. Osteoporos Int 1998; 8: 75-81.

29. Gnudi S, Ripamonti C, Lisi L, Fini M, Giardino R, Giavaresi G. Proximal femur geometry to detect and distinguish femoral neck fractures from trochanteric fractures in postmenopausal women. Osteoporos Int 2002; 13: 69-73.

30. Johnell O, Kanis JA, Oden A, et al. Predictive value of BMD for hip and other fractures. J Bone Miner Res 2005; 20: 1185-94.

31. Li Y, Lin J, Cai S, et al. Influence of bone mineral density and hip geometry on the different types of hip fracture. Bosn J Basic Med Sci 2016; 16: 35-8. 